\title{
Low vaspin levels are related to endothelial dysfunction in patients with ankylosing spondylitis
}

\author{
H.H. Wang ${ }^{1}$ and Q.F. Wang ${ }^{2}$ \\ ${ }^{1}$ Dongguan People's Hospital, Dongguan, Guangdong Province, China \\ ${ }^{2}$ Department of Rheumatology, PAL Hospital, Beijing, China
}

\begin{abstract}
Vaspin is a novel adipocytokine associated with glucose tolerance and chronic inflammation. Some studies reveal that vaspin may be involved in cardiovascular diseases. Our objective was to investigate the relationship between serum vaspin levels and endothelial function in patients with ankylosing spondylitis. One hundred and twenty patients with newly diagnosed ankylosing spondylitis and 100 healthy subjects were studied. Serum vaspin levels were measured with enzyme-linked immunosorbent assay. High resolution ultrasound was used to measure brachial artery diameter at rest, after reactive hyperemia (flow-mediated dilation, FMD) and after sublingual glyceryltrinitrate. Serum vaspin level in patients was $1.92 \pm 1.03 \mathrm{ng} / \mathrm{mL}$, which was significantly lower than that in healthy subjects $(2.88 \pm 0.81 \mathrm{ng} / \mathrm{mL})$. By dividing the distribution of serum vaspin levels into quartiles, FMD levels increased gradually with the increase of serum vaspin levels in patients $(P<0.01)$. Univariate analysis showed a correlation between vaspin and FMD ( $r=0.73, P=0.003)$, low-density lipoprotein cholesterol $(r=-0.45, P=0.033)$, highdensity lipoprotein cholesterol $(r=0.63, P=0.025)$, fasting blood glucose $(r=-0.79, P=0.006)$, triglycerides (TG) ( $r=-0.68$, $\mathrm{P}=0.036)$, systolic blood pressure $(r=-0.35, P=0.021)$, C-reactive protein $(r=-0.67, P=0.011)$, homeostatic model assessment of insulin resistance (HOMA-IR) $(r=-0.77, P=0.023)$ and erythrocyte sedimentation rate $(r=-0.88, P=0.039)$ in patients. Multivariate analysis indicated that serum vaspin levels were independently associated with FMD, HOMA-IR and TG in patients. Our study found that serum vaspin levels were decreased in patients with ankylosing spondylitis and were associated with FMD levels. Vaspin may serve as an independent marker for detecting early stage atherosclerosis in patients with ankylosing spondylitis.
\end{abstract}

Key words: Vaspin; Endothelial function; Ankylosing spondylitis

\section{Introduction}

Vaspin is recognized as a novel adipokine in the modulation of adipocyte differentiation and glucose homeostasis $(1,2)$. It has been proven to be a serine protease inhibitor family, which is associated with insulin resistance and metabolic syndrome (3). Furthermore, serum vaspin levels are associated with inflammation in rheumatoid arthritis $(4,5)$. Recently, some studies have shown that vaspin has been linked to atherosclerosis and cardiovascular disease $(6,7)$. Insulin resistance (IR) and chronic inflammation play an important role in the development of cardiovascular diseases $(8,9)$. It has been assumed that vaspin serves as a potential insulin-sensitizing agent with anti-inflammatory effects and might act as a compensatory mechanism in the progression of atherosclerosis.

Endothelial dysfunction is an early event of atherosclerosis and plays a central role in the development and progression of cardiovascular diseases (10). Ankylosing spondylitis is associated with accelerated atherosclerosis and increased risk of metabolic syndrome (11-13). Clinical studies have shown that the impairment of flowmediated dilation (FMD) is present in patients with ankylosing spondylitis (14-16). Sari et al., in a study where they excluded patients who had common cardiovascular risk factors, reported that ankylosing spondylitis had a role in the development of endothelial impairment (15). On the other hand, endothelial function of ankylosing spondylitis patients was impaired compared with healthy controls, and improved after tumor necrosis factor-alpha blockade (16).

Since no data is yet available on the relationship between serum vaspin levels and endothelial function in patients with ankylosing spondylitis, we tested the hypothesis that vaspin levels are associated with early stage atherosclerosis in patients with ankylosing spondylitis. The purpose of this study was to evaluate the relationship between vaspin and endothelial fucntion in patients with ankylosing spondylitis. 


\section{Subjects and Methods}

\section{Subjects}

From January 2013 to September 2015, 120 newly diagnosed patients with ankylosing spondylitis and 100 age-matched healthy volunteers (controls) were enrolled in our study. The diagnosis of ankylosing spondylitis was based on the modified New York criteria (17). Exclusion criteria were set according to previous studies by our group $(18,19)$. All participants who presented a history of congestive heart failure, myocardial infarction or angina were excluded. Besides, obese people (BMI $\left.>30 \mathrm{~kg} / \mathrm{m}^{2}\right)$, and people with malignant neoplasms, hypertension, renal or liver diseases were also excluded from our study. In addition, patients could not be taking any drugs, such as thyroxine, estrogen supplements, diuretics, antihypertensive or $\beta$-blockers, or lipid-lowering drugs. The study protocol was in agreement with the ethics guidelines of the committee at Dongguan People's Hospital, and was performed in accordance with the 1964 Declaration of Helsinki. Written consent or a thumb-print was required from all participants before screening.

\section{Methods}

Biochemical measurements. The parameters were evaluated as described previously $(18,19)$. Venous blood samples were drawn after a 12- to 14-h overnight fast. The serum vaspin levels were measured using a human vaspin sandwich enzyme-linked immunosorbent assay. Serum samples were diluted to $1 / 3$ and measured in duplicate, and the results were averaged. The intra-assay coefficient of variation was $5-5.5 \%$.

Total cholesterol (TC), triglycerides (TG), high-density lipoprotein cholesterol (HDL-C), and low-density lipoprotein cholesterol (LDL-C), were enzymatically determined. The fasting blood glucose (FBG) was analyzed by a glucose oxidase method. Levels of ultrasensitive C-reactive protein (CRP) levels were analyzed by immunoturbidimetric analysis. Erythrocyte sedimentation rate (ESR) and creatinine were determined using an automated analyzer (Sysmex XE5000, Japan). Coefficients of variation for these assays were 1-2\% (TC, glucose, HDL-C), $1-2 \%$ (LDL-C, TG, CRP), and 1-3\% (ESR).

Ultrasound examination of the brachial artery. Vascular examination of the brachial artery was performed noninvasively, as described previously $(18,19)$. High resolution ultrasound was used to measure changes in arterial diameter in response to reactive hyperemia (with increased flow producing an endothelium-dependent stimulus to vasodilatation, FMD), and to glyceryltrinitrate (GTN-induced endothelium-independent arterial dilation; $128 \mathrm{XP} / 10$ with a $7.0-\mathrm{MHz}$ linear array transducer; Acuson, USA). The intra- and inter-observer variability in our laboratory for repeated measurements of artery diameter is $0.09 \pm 0.10$ and $0.08 \pm 0.13 \mathrm{~mm}$, respectively.

\section{Statistical analysis}

To compare numerical variables between two groups we used unpaired Student's t-test. The differences between categorical variables or nominal variables were analyzed with chi-square test. Univariate analysis was used for basic characteristics of participants expressed as categorical and continuous variables, including (FMD, vessel size, blood flow, GTN-induced endothelium-independent arterial dilation, LDL-C, HDL-C, TC, HOMA-IR, TG, CRP, diastolic and systolic blood pressure (DBP and SBP), FBG, age, ESR and BMI), while one-way analysis of variance (ANOVA) was used for categorical variables (smoking, family history and gender). Multiple linear regression analysis was performed to evaluate the association between vaspin and other independent variables. Pearson's correlation coefficients were calculated to determine the correlation between variables. Patient were stratified into four quartiles by vaspin levels. The between-group differences were evaluated by ANOVA. Significance was accepted at $P<0.05$. All calculations were performed by SPSS 13.0 (IBM, USA).

\section{Results}

Table 1 shows the clinical characteristics of the control subjects and patients. Serum vaspin levels were significantly lower in patients with ankylosing spondylitis compared with the controls $(1.92 \pm 1.03$ vs $2.88 \pm 0.81$ $\mathrm{ng} / \mathrm{mL}, \quad \mathrm{P}<0.001)$. Ankylosing spondylitis subjects showed higher HOMA-IR, ESR, TG and CRP levels, compared with control group $(P<0.01)$.

Patients were categorized into quartiles of serum vaspin levels (Table 2). These four groups did not show any difference in gender, age, BMI, positive family history, FBG, smoker, SBP, DBP, HDL-C, creatinine, baseline vessel, baseline flow and GTN-induced endothelium-independent arterial dilation $(P>0.05)$. TG, CRP, HOMA-IR and ESR were gradually lower with higher serum vaspin levels $(P<0.05)$. However, the FMD levels were gradually higher with higher serum vaspin levels $(P<0.05)$. LDL-C and TC showed a tendency to be lower when serum vaspin levels were gradually higher $(P>0.05)$.

Univariate analysis between serum vaspin levels and variables such as FMD $(r=0.73, P=0.003)$, LDL-C $(r=-0.45, P=0.033)$, HDL-C $(r=0.63, P=0.025)$, FBG $(r=$ $-0.79, P=0.006), \mathrm{TG}(r=-0.68, P=0.036)$, SBP $(r=-0.35$, $P=0.021), \operatorname{CRP}(r=-0.67, P=0.011)$, HOMA-IR $(r=-0.77$, $P=0.023)$ and $E S R(r=-0.88, P=0.039)$ showed significant correlations $(P<0.05)$. By multivariate regression analysis, FMD, LDL-C, HDL-C, FBG, TG, SBP, CRP, HOMA-IR, and ESR were independently associated to serum vaspin levels in patients with ankylosing spondylitis. The significant determinants of serum vaspin levels were FMD, HOMA-IR and TG. Pearson's analysis showed a significant association between serum vaspin levels and FMD levels $(P<0.05$; Figure 1$)$. 
Table 1. Clinical and biochemical characteristics in healthy control subjects and patients with ankylosing spondylitis.

\begin{tabular}{|c|c|c|c|}
\hline Variables & $\begin{array}{l}\text { Control } \\
\text { subjects }\end{array}$ & $\begin{array}{c}\text { Patients with ankylosing } \\
\text { spondylitis }\end{array}$ & $\mathrm{P}$ \\
\hline Number of subjects & 100 & 120 & - \\
\hline Age (years) & $56 \pm 12$ & $60 \pm 13$ & 0.77 \\
\hline Gender (M/F) & $55 / 45$ & $68 / 52$ & 0.28 \\
\hline Positive family history, n (\%) & $2(2)$ & $12(10)$ & - \\
\hline Smoker, n (\%) & $8(8)$ & $15(12.5)$ & - \\
\hline $\mathrm{SBP}(\mathrm{mmHg})$ & $116.8 \pm 5.82$ & $138.3 \pm 5.93$ & 0.089 \\
\hline $\mathrm{DBP}(\mathrm{mmHg})$ & $75.6 \pm 12.93$ & $85.3 \pm 15.85$ & 0.111 \\
\hline BMI $\left(\mathrm{kg} / \mathrm{m}^{2}\right)$ & $23.1 \pm 6.6$ & $25.1 \pm 5.5$ & 0.89 \\
\hline FBG (mmol/L) & $4.51 \pm 2.13$ & $5.52 \pm 1.33$ & 0.31 \\
\hline $\operatorname{ESR}(\mathrm{mm} / \mathrm{h})$ & $3.0 \pm 1.81$ & $12.12 \pm 2.15$ & $<0.001$ \\
\hline $\mathrm{TC}(\mathrm{mmol} / \mathrm{L})$ & $4.11 \pm 1.29$ & $4.15 \pm 1.85$ & 0.128 \\
\hline LDL-C (mmol/L) & $2.31 \pm 0.67$ & $2.12 \pm 0.31$ & 0.241 \\
\hline $\mathrm{HDL}-\mathrm{C}(\mathrm{mmol} / \mathrm{L})$ & $1.51 \pm 0.85$ & $1.0 \pm 0.13$ & 0.001 \\
\hline TG $(\mathrm{mmol} / \mathrm{L})$ & $1.32 \pm 0.79$ & $1.98 \pm 1.01$ & 0.005 \\
\hline HOMA-IR & $2.33 \pm 1.06$ & $2.93 \pm 1.13$ & $<0.001$ \\
\hline Creatinine ( $\mu \mathrm{mol} / \mathrm{L})$ & $75.99 \pm 21.35$ & $82.95 \pm 18.33$ & 0.299 \\
\hline CRP (mg/L) & $1.09 \pm 0.55$ & $2.99 \pm 0.77$ & $<0.001$ \\
\hline Vaspin (ng/mL) & $2.88 \pm 0.81$ & $1.92 \pm 1.03$ & $<0.001$ \\
\hline
\end{tabular}

Data are reported as means \pm SD, unless otherwise indicated. SBP: systolic blood pressure; DBP: diastolic blood pressure; BMI: body mass index; FBG: fasting blood glucose; ESR: erythrocyte sedimentation rate; TC: total cholesterol; LDL-C: low-density lipoprotein cholesterol; HDL-C: high-density lipoprotein cholesterol; TG: triglycerides; HOMA-IR: homeostatic model assessment of insulin resistance; CRP: C-reactive protein. Data analysis of categorical variables was performed with the chi-square test and of continuous variables with the $t$-test.

\section{Discussion}

Direct evidence of the role of vaspin in endothelial function is lacking, especially in patients with ankylosing spondylitis. We found that serum vaspin levels are related with endothelial function, and significantly decreased in patients with ankylosing spondylitis, compared to controls. Our study indicated that vaspin might be employed as a novel prognostic marker in patients with ankylosing spondylitis.

Vaspin, containing 415 amino acids secreted by visceral adipose tissue, was reported to modulate different functions as a ligand for a cell-surface receptor complex (1). Nakatsuka et al. have demonstrated that vaspintransgenic mice are protected against diet-induced obesity, glucose tolerance impairment and fatty liver (20). Administration of recombinant vaspin improves glucose intolerance, and insulin sensitivity in diet-induced obese mice (21). Vaspin alleviates the dysfunction of endothelial progenitor cells induced by high glucose (22). In addition, a previous study reported that vaspin inhibited the progression of atherosclerotic plaques in apoE ${ }^{-1-}$ mice by inhibiting endoplasmic reticulum stress-induced macrophage apoptosis (23). Increased vaspin expression may be an intrinsic compensatory mechanism in adipose tissue as a response to decreased insulin sensitivity or impairment of glucose metabolism (24). Vaspin levels were found to have an inverse relationship with the risk of cardiovascular events, suggesting a protective role of vaspin in the pathophysiology of coronary atherosclerosis (25). More recently, clinical studies have shown that vaspin was associated with insulin resistance, chronic inflammation and might be involved in the development of metabolic diseases (3). Our study reports the new adipokine vaspin in ankylosing spondylitis, and it shows that vaspin is significantly lower in ankylosing spondylitis compared with healthy controls. The mechanism related with lower vaspin levels was not investigated in our study. Since vaspin also exerts anti-inflammatory properties, like the suppression of TNFalpha (26), it would be conceivable that lower vaspin levels might be a consequence of the increase of pro-inflammatory cytokines in ankylosing spondylitis. In the present study, vaspin was correlated with CRP and ESR, which are in agreement with this hypothesis.

Much controversy exists about the relationship of vaspin with different chronic inflammatory diseases. 
Table 2. Clinical and biochemical characteristics of patients with ankylosing spondylitis according to the quartiles of serum vaspin levels.

\begin{tabular}{lccccc}
\hline \multirow{2}{*}{ Variables } & \multicolumn{3}{c}{ Quartiles of serum vaspin levels } & P value \\
\cline { 2 - 4 } & Quartile 1 & Quartile 2 & Quartile 3 & Quartile 4 & for trend \\
\hline Number of subjects & 30 & 30 & 30 & 30 \\
Age (years) & $68 \pm 13$ & $62 \pm 11$ & $53 \pm 18$ & $58 \pm 5$ & 0.169 \\
BMI (kg/m ${ }^{2}$ ) & $26.54 \pm 2.56$ & $19.33 \pm 3.52$ & $23.11 \pm 3.01$ & $22.56 \pm 1.11$ & 0.231 \\
Gender (M/F) & $15 / 15$ & $16 / 14$ & $23 / 7$ & $15 / 15$ & 0.785 \\
Positive family history, n (\%) & $6(20)$ & $4(13.3)$ & $1(3.3)$ & $1(3.3)$ & 0.088 \\
Smoker, $\mathrm{n}(\%)$ & $6(20)$ & $3(10)$ & $5(17)$ & $3(10)$ & 0.111 \\
SBP (mmHg) & $153.1 \pm 9.3$ & $137.9 \pm 10.2$ & $145.5 \pm 12.2$ & $128.2 \pm 13.1$ & 0.841 \\
DBP (mmHg) & $80.3 \pm 20.5$ & $85.6 \pm 19.8$ & $95.3 \pm 18.1$ & $73.3 \pm 16.9$ & 0.697 \\
FBG (mmol/L) & $8.12 \pm 3.11$ & $6.51 \pm 3.52$ & $4.34 \pm 2.39$ & $5.61 \pm 1.33$ & 0.123 \\
ESR (mmol/L) & $19.58 \pm 3.115$ & $13.55 \pm 1.66$ & $8.88 \pm 2.55$ & $5.77 \pm 1.99$ & 0.000 \\
TC (mmol/L) & $4.95 \pm 0.52$ & $4.10 \pm 1.88$ & $3.88 \pm 1.80$ & $2.55 \pm 0.77$ & 0.185 \\
LDL-C (mmol/L) & $3.00 \pm 0.12$ & $2.12 \pm 1.05$ & $2.18 \pm 0.56$ & $1.62 \pm 0.85$ & 0.652 \\
HDL-C (mmol/L) & $0.49 \pm 0.17$ & $0.98 \pm 0.22$ & $1.23 \pm 0.85$ & $1.37 \pm 0.69$ & 0.133 \\
TG (mmol/L) & $2.85 \pm 0.95$ & $1.75 \pm 1.11$ & $1.21 \pm 0.63$ & $1.01 \pm 0.99$ & 0.010 \\
HOMA-IR & $4.11 \pm 1.01$ & $3.91 \pm 0.55$ & $3.01 \pm 0.96$ & $2.08 \pm 0.81$ & 0.000 \\
Creatinine ( $\mu$ mol/L) & $85.11 \pm 10.77$ & $88.87 \pm 25.85$ & $95.56 \pm 16.21$ & $77.15 \pm 15.75$ & 0.985 \\
CRP (mg/L) & $3.31 \pm 1.78$ & $2.79 \pm 0.89$ & $1.98 \pm 1.31$ & $1.31 \pm 0.92$ & 0.000 \\
FMD (\%) & $2.65 \pm 0.85$ & $3.65 \pm 0.59$ & $4.28 \pm 0.78$ & $4.99 \pm 0.29$ & 0.009 \\
Baseline vessel (mm) & $4.00 \pm 0.33$ & $3.88 \pm 0.45$ & $3.01 \pm 0.43$ & $4.85 \pm 0.55$ & 0.253 \\
Baseline flow (mL/min) & $88.17 \pm 13.11$ & $90.12 \pm 25.22$ & $96.18 \pm 23.55$ & $75.70 \pm 20.89$ & 0.415 \\
GTN-induced dilation (\%) & $20.68 \pm 8.55$ & $30.18 \pm 3.55$ & $15.10 \pm 9.11$ & $29.55 \pm 7.10$ & 0.898 \\
\hline
\end{tabular}

Data are reported as means \pm SD, unless otherwise indicated. SBP: systolic blood pressure; DBP: diastolic blood pressure; BMI: body mass index; FBG: fasting blood glucose; ESR: erythrocyte sedimentation rate; TC: total cholesterol; LDL-C: low-density lipoprotein cholesterol; HDL-C: high-density lipoprotein cholesterol; TG: triglycerides; HOMA-IR: homeostatic model assessment of insulin resistance; CRP: C-reactive protein; FMD: flow-mediated dilation; GTN: glyceryltrinitrate. Data analysis was performed with ANOVA.

Morisaki et al. (27) have shown that vaspin plasma levels are elevated in patients with ulcerative colitis and increase further after remission induction. The study by Ozgen et al. (5) shows an important association between vaspin and chronic inflammatory diseases. They reported that vaspin levels are higher in rheumatoid arthritis and may be involved in the regulation of inflammatory responses in inflammatory diseases. Moreover, in contrast to rheumatoid arthritis, vaspin levels decline in active Behcet's disease. The above studies suggest that different chronic inflammatory diseases exert different influences on vaspin levels. Thus, the relationship between vaspin and different chronic inflammatory diseases needs to be confirmed by further studies with larger number of subjects.

Numerous studies have reported on the association of vaspin with cardiovascular diseases $(6,7)$. It has been reported that vaspin concentrations are associated with coronary atherosclerosis (7). Esaki et al. (6) published an observational study among 201 subjects from the general population in Japan, where they retrospectively measured vaspin levels. They found an independent association between serum vaspin levels and intima-media thickness. Moreover, previous works have suggested that low vaspin emerged as an important factor for coronary artery disease (28). Endothelial dysfunction is one of the first signs of atherosclerosis and cardiovascular diseases. Brachial FMD has been a widely used noninvasive method for the assessment of vascular endothelial function. Many clinical studies showed that ankylosing spondylitis is associated with vascular endothelial dysfunction, which leads to accelerated atherosclerosis. However, few studies have evaluated the association of vaspin with endothelial function in patients with ankylosing spondylitis. The present study found that vaspin was associated with endothelial function, in which serum vaspin levels in patients increased gradually as FMD levels increased from quartile 1 to quartile 4 (Table 2).

Metabolic syndromes are considered diseases with common traits that can increase the risk of cardiovascular disease (29). Ankylosing spondylitis is a chronic inflammatory disease associated with a metabolic syndrome (30). 


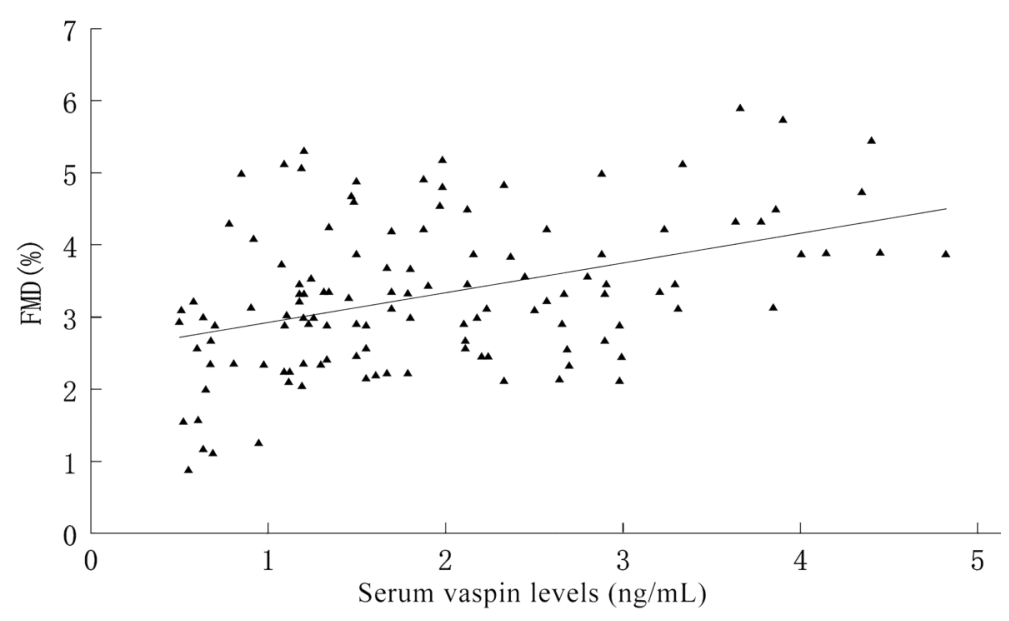

Figure 1. Correlation between serum vaspin levels and flow-mediated dilation (FMD) in patients with ankylosing spondylitis.
In current clinical practice, we found that serum vaspin levels were negatively correlated with TG, CRP and HOMA$I R$, which is in agreement with a previous study (31). In addition, LDL-C and TC levels decreased when serum vaspin levels increased gradually.

The effects of vaspin on endothelial function as well as the regulatory mechanisms in ankylosing spondylitis are poorly understood. FMD is a proposed indicator of nitric oxide-bioavailability and vascular function (10). Vaspin increases nitric oxide bioavailability through the reduction of asymmetric dimethylarginine in vascular endothelial cells (32). The study by Sun et al. (22) indicated a novel effect of vaspin to regulate eNOS expression and function in endothelial progenitor cells via a PI3K/Akt/eNOS pathway, and vaspin may have a protective effect in patients with diabetes in preventing the occurrence of vascular complications. Further studies are needed to explain the mechanism by which vaspin relates with endothelial function in ankylosing spondylitis.

\section{References}

1. Hida K, Wada J, Eguchi J, Zhang H, Baba M, Seida A, et al. Visceral adipose tissue-derived serine protease inhibitor: a unique insulin-sensitizing adipocytokine in obesity. Proc Natl Acad Sci U S A 2005; 102: 10610-10615, doi: 10.1073/ pnas. 0504703102 .

2. Wada J. Vaspin: a novel serpin with insulin-sensitizing effects. Expert Opin Investig Drugs 2008; 17: 327-333, doi: 10.1517/13543784.17.3.327.

3. Dimova R, Tankova T. The role of vaspin in the development of metabolic and glucose tolerance disorders and atherosclerosis. Biomed Res Int 2015; 2015: 823481, doi: $10.1155 / 2015 / 823481$.

4. Klaasen R, Herenius MM, Wijbrandts CA, de Jager W, van Tuyl LH, Nurmohamed MT, et al. Treatment-specific changes in circulating adipocytokines: a comparison between tumour necrosis factor blockade and glucocorticoid
The most significant limitation of this study is the small sample size and we can not exclude the effect of other adipokines such as irisin, chemerin and adiponectin. Besides, the corresponding levels of cell-surface receptor complex was not evaluated. Another limitation is that intraobserver variation was not assessed in this study.

In conclusion, this study showed that serum vaspin levels were decreased in patients with ankylosing spondylitis, and were significantly associated with endothelial function. The mechanism for the relationship between vaspin and endothelial function in ankylosing spondylitis needs to be explored in the future.

\section{Acknowledgments}

This research was supported by Dongguan People's Hospital. The authors would like to thank Professor Guangda Xiang for excellent technical support and for critically revising the manuscript.

treatment for rheumatoid arthritis. Ann Rheum Dis 2012; 71: 1510-1516, doi: 10.1136/annrheumdis-2011-200646.

5. Ozgen M, Koca SS, Dagli N, Balin M, Ustundag B, Isik A. Serum adiponectin and vaspin levels in rheumatoid arthritis. Arch Med Res 2010; 41: 457-463, doi: 10.1016/j.arcmed. 2010.08.012.

6. Esaki E, Adachi H, Hirai Y, Yamagishi S, Kakuma T, Enomoto $\mathrm{M}$, et al. Serum vaspin levels are positively associated with carotid atherosclerosis in a general population. Atherosclerosis 2014; 233: 248-252, doi: 10.1016/ j.atherosclerosis.2013.12.040

7. Choi SH, Kwak SH, Lee Y, Moon MK, Lim S, Park YJ, et al. Plasma vaspin concentrations are elevated in metabolic syndrome in men and are correlated with coronary atherosclerosis in women. Clin Endocrinol 2011; 75: 628-635, doi: 10.1111/j.1365-2265.2011.04095.x. 
8. Castellon X, Bogdanova V. Chronic inflammatory diseases and endothelial dysfunction. Aging Dis 2016; 7: 81-89, doi: 10.14336/AD.2015.0803.

9. Babu A, Fogelfeld L. Metabolic syndrome and prediabetes. Dis Mon 2006; 52: 55-144, doi: 10.1016/j.disamonth.2006.03.001.

10. Celermajer DS, Sorensen KE, Gooch VM, Spiegelhalter DJ, Miller OI, Sullivan ID, et al. Non-invasive detection of endothelial dysfunction in children and adults at risk of atherosclerosis. Lancet 1992; 340: 1111-1115, doi: 10.1016/01406736(92)93147-F.

11. El Maghraoui A. Extra-articular manifestations of ankylosing spondylitis: prevalence, characteristics and therapeutic implications. Eur J Intern Med 2011; 22: 554-560, doi: 10.1016/j.ejim.2011.06.006.

12. Gonzalez-Juanatey C, Vazquez-Rodriguez TR, MirandaFilloy JA, Dierssen T, Vaqueiro I, Blanco R, et al. The high prevalence of subclinical atherosclerosis in patients with ankylosing spondylitis without clinically evident cardiovascular disease. Medicine 2009; 88: 358-365, doi: 10.1097/ MD.0b013e3181c10773.

13. Papadakis JA, Sidiropoulos PI, Karvounaris SA, Vrentzos GE, Spanakis EK, Ganotakis ES, et al. High prevalence of metabolic syndrome and cardiovascular risk factors in men with ankylosing spondylitis on anti-TNFalpha treatment: correlation with disease activity. Clin Exp Rheumatol 2009; 27: 292-298.

14. Syngle A, Vohra K, Khichi D, Garg N, Verma I, Kaur L. Spironolactone improves endothelial dysfunction in ankylosing spondylitis. Clin Rheumatol 2013; 32: 1029-1036, doi: 10.1007/s10067-013-2233-3.

15. Sari I, Okan T, Akar S, Cece H, Altay C, Secil M, et al. Impaired endothelial function in patients with ankylosing spondylitis. Rheumatology 2006; 45: 283-286, doi: 10.1093/ rheumatology/kei145.

16. Syngle A, Vohra K, Sharma A, Kaur L. Endothelial dysfunction in ankylosing spondylitis improves after tumor necrosis factor-alpha blockade. Clin Rheumatol 2010; 29 : 763-770, doi: 10.1007/s10067-010-1402-x.

17. van der Linden S, Valkenburg HA, Cats A. Evaluation of diagnostic criteria for ankylosing spondylitis. A proposal for modification of the New York criteria. Arthritis Rheum 1984; 27: 361-368, doi: 10.1002/art.1780270401.

18. Wang $\mathrm{HH}$, Zhang XW, Chen WK, Huang QX, Chen QQ. Relationship between serum irisin levels and urinary albumin excretion in patients with type 2 diabetes. J Diabetes Complications 2015; 29: 384-389, doi: 10.1016/j.jdiacomp.2015.01.001.

19. Wang HH, Xiang GD. Changes of plasma concentration of osteoprotegerin and its association with endothelial dysfunction before and after hypouricemic therapy in patients with hyperuricemia. Mod Rheumatol 2015; 25: 123-127, doi: 10.3109/14397595.2014.926852.

20. Nakatsuka A, Wada J, Iseda I, Teshigawara S, Higashio K, Murakami K, et al. Visceral adipose tissue-derived serine proteinase inhibitor inhibits apoptosis of endothelial cells as a ligand for the cell-surface GRP78/voltage-dependent anion channel complex. Circ Res 2013; 112: 771-780, doi: $10.1161 /$ CIRCRESAHA.111.300049.

21. Wada J. [Vaspin and insulin resistance]. Rinsho Byori 2008; 56: 705-711.

22. Sun $\mathrm{N}$, Wang $\mathrm{H}$, Wang L. Vaspin alleviates dysfunction of endothelial progenitor cells induced by high glucose via PI3K/Akt/eNOS pathway. Int J Clin Exp Pathol 2015; 8: 482-489.

23. Lin Y, Zhuang J, Li H, Zhu G, Zhou S, Li W, et al. Vaspin attenuates the progression of atherosclerosis by inhibiting ER stressinduced macrophage apoptosis in apoE/ mice. Mol Med Rep 2016; 13: 1509-1516, doi: 10.3892/mmr.2015. 4708.

24. Kloting N, Berndt J, Kralisch S, Kovacs P, Fasshauer M, Schon MR, et al. Vaspin gene expression in human adipose tissue: association with obesity and type 2 diabetes. Biochem Biophys Res Commun 2006; 339: 430-436, doi: 10.1016/j.bbrc.2005.11.039.

25. Kadoglou NP, Gkontopoulos A, Kapelouzou A, Fotiadis G, Theofilogiannakos EK, Kottas G, et al. Serum levels of vaspin and visfatin in patients with coronary artery diseaseKozani study. Clin Chim Acta 2011; 412: 48-52, doi: 10.1016/j.cca.2010.09.012.

26. Hida K, Wada J, Eguchi J, Zhang H, Baba M, Seida A, et al. Visceral adipose tissue-derived serine protease inhibitor: a unique insulin-sensitizing adipocytokine in obesity. Proc Natl Acad Sci U S A 2005; 102: 10610-10615, doi: 10.1073/ pnas.0504703102.

27. Morisaki T, Takeshima F, Fukuda H, Matsushima K, Akazawa $\mathrm{Y}$, Yamaguchi $\mathrm{N}$, et al. High serum vaspin concentrations in patients with ulcerative colitis. Dig Dis Sci 2014; 59: 315-321, doi: 10.1007/s10620-0132905-5.

28. Karbek B, Bozkurt NC, Topaloglu O, Aslan MS, Gungunes A, Cakal E, et al. Relationship of vaspin and apelin levels with insulin resistance and atherosclerosis in metabolic syndrome. Minerva Endocrinol 2014; 39: 99-105.

29. Babu A, Fogelfeld L. Metabolic syndrome and prediabetes. Dis Mon 2006; 52: 55-144, doi: 10.1016/j.disamonth.2006. 03.001.

30. Papadakis JA, Sidiropoulos PI, Karvounaris SA, Vrentzos GE, Spanakis EK, Ganotakis ES, et al. High prevalence of metabolic syndrome and cardiovascular risk factors in men with ankylosing spondylitis on anti-TNFalpha treatment: correlation with disease activity. Clin Exp Rheumatol 2009; 27: 292-298.

31. Choi SH. Letter: association of vaspin with metabolic syndrome: the pivotal role of insulin resistance (diabetes metab j 2014;38:143-9). Diabetes Metab J 2014; 38: 240-241, doi: 10.4093/dmj.2014.38.3.240.

32. Jung CH, Lee WJ, Hwang JY, Lee MJ, Seol SM, Kim YM, et al. Vaspin increases nitric oxide bioavailability through the reduction of asymmetric dimethylarginine in vascular endothelial cells. PLoS One 2012; 7: e52346, doi: 10.1371/ journal.pone.0052346. 